\title{
THE SPACE GEODESY PROJECT AND RADIO FREQUENCY INTERFERENCE CHARACTERIZATION AND MITIGATION
}

\author{
Hilliard, Lawrence M., ${ }^{2}$ Beaudoin, C., ${ }^{2}$ Corey, B.E., ${ }^{3}$ Tourain, C., L, ${ }^{4}$ Petrachenko, B, ${ }^{5}$ Dickey, John \\ ${ }^{1}$ NASA Goddard Space Flight Center, Greenbelt MD, USA \\ ${ }^{2}$ MIT Haystack Observatory, Westford MA, USA \\ ${ }^{3}$ Centre National d'Etudes Spatiales (CNES), Toulouse France \\ ${ }^{4}$ National Research Council Canada \\ ${ }^{5}$ University of Tasmania, Australia
}

Abstract - The Space Geodesy Project (SGP) development by NASA is an effort to co-locate the four international geodetic techniques Satellite Laser Ranging (SLR) and Lunar Laser Ranging (LLR), Very Long Baseline Interferometry (VLBI), Global Navigation Satellite System (GNSS), and Doppler Orbitography and Radiopositioning Integrated by Satellite (DORIS) into one tightly referenced campus and coordinated reference frame analysis. The SGP requirement locates these stations within a small area to maintain line-of-sight and frequent automated survey known as the vector tie system. This causes a direct conflict with the new broadband VLBI technique. Broadband means $2-14 \mathrm{GHz}$, and RFI susceptibility at $-80 \mathrm{dBW}$ or higher due to sensitive $\mathrm{RF}$ components in the front end of the radio receiver.

\section{SGP INTRODUCTION}

The goals of the Space Geodesy Project are as follows:

Establish and operate a prototype next generation space geodetic station with integrated next generation SLR, VLBI, GNSS (and DORIS) systems, along with a system that provides for accurate vector ties between them.

Develop a Project Implementation Plan for the construction, deployment and operation of a NASA network of similar next generation stations that will become the core of a larger global network of modern space geodetic stations.

Demonstration by August 2013.

The key integrator of the four techniques, the vector tie system, must be near enough to validate automated survey techniques with traditional ones, Vector Tie System (VTS)

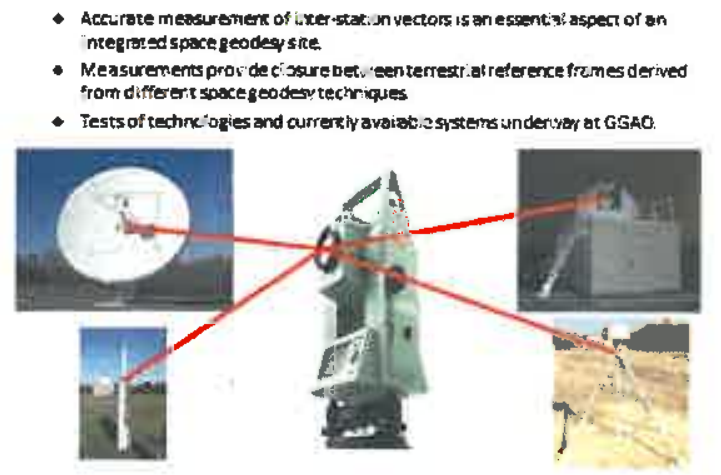

So there we have competing requirements between the vector tie system and the VLBI 2010 prototype that is specially designed to tolerate high radio frequency interference environments. 


\section{VLBI 2010}

The reasons that VLBI developers have adopted the wide bandwidth approach are twofold. First, additional data must be taken faster to better sample the celestial reference frame (CRF) measured from the group delay of quasars arriving at the Earth as plane waves. To calibrate out delay uncertainties caused by local weather and pressure and the ionosphere across long baselines, the plane waves must be analyzed to 10 times the precision of its legacy network, which is 4 picoseconds, or a $1 \mathrm{~mm}$ in distance. Although the technique has been used since the 1970's, the International VLBI Service for Geodesy and Astrometry (IVS) working group created a vision for the future in the early 2000 's by establishing the VLBI 2010 concept.

A description of the VLBI 2010 prototype under development at the Goddard Geophysical and Astronomical Observatory (GGAO) at NASA's Goddard Space Flight Center is described in figure 1.

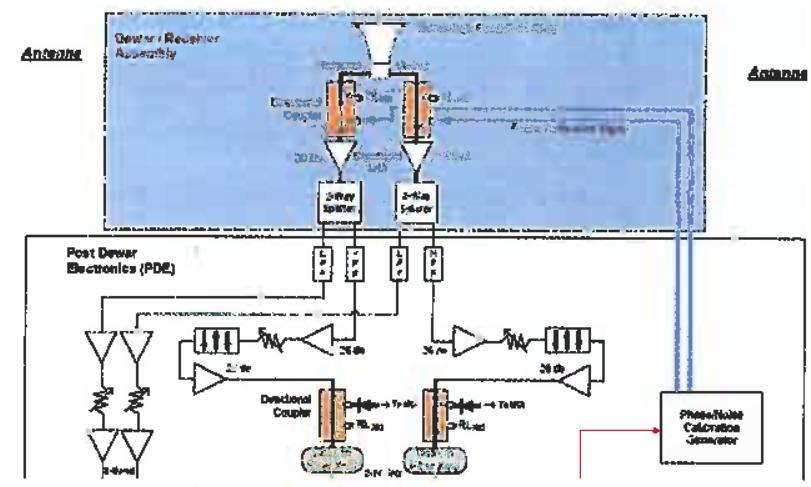

Figure 1: $A$ high pass and low pass filter isolate the prominent S-Band RFI at GGAO

New technologies in this ground system include the Quadridge flared horn (QRFH) developed for Haystack Observatory by the California Institute of
Technology. Low noise amplifiers that operate cryogenically at very low noise temperatures are also included in VLBI 2010. Although a very agile antenna that VLBI 2010 is using at GGAO has been in warm operation at Australian stations Hobart and Yarragadee, the NASA funded 12 meter antenna is the $1^{\text {st }}$ attempt at cryogenic operation, and many lessons have been learned on the GGAO prototype slewing at 5 degrees per second in azimuth. The elevation slewing is slower in the prototype, but the rapid target acquisition and collection of data on a target has been demonstrated in three 6-hour geodetic baselines between Westford, Massachusetts and GGAO. The other reason to adopt the wide bandwidth is to provide the maximum flexibility for avoiding sources of radio frequency interference (RFI).

\section{SLR RFI -RADARS}

Most inconvenient are the legacy VLBI channels and the RFI emanating due to SLR Laser Hazard Ranging System (LHRS) and DORIS beacon. The lasers themselves do not use microwave frequencies, but it is an air traffic safety requirement to have radar that detects an airplane in the vicinity of the laser to automatically shut down the laser. That radar is at 9.4 $\mathrm{GHz}$ for both the Next Generation SLR and the operational Mobile Laser System (MobLas7), both operating at GGAO. Location of these geodetic techniques near each other causes a sky coverage limitation, to the south for VLBI, and to the north for the two SLR systems.

With excellent cooperation between the development teams we have quantified the effects of masking the sky, and characterized the susceptibility of the 12 
meter antenna over a full raster scan with a test beacon

(Figure 2 shows both the avoidance mask for both SLR radars).

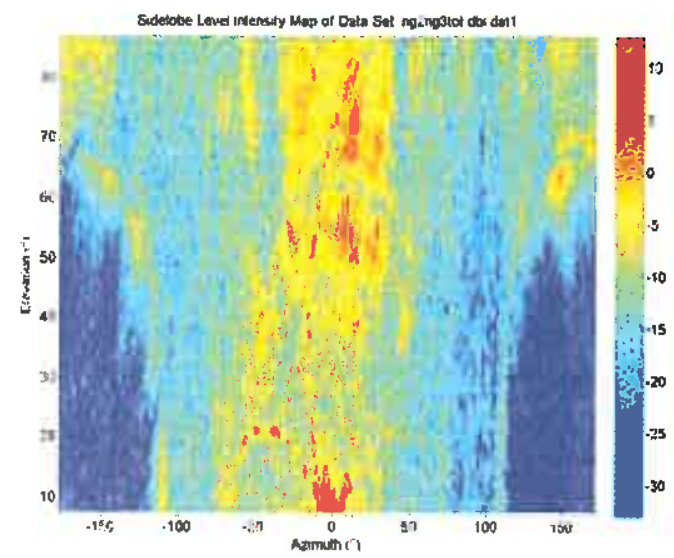

Figure 2: The sidelobes of the 12 meter in response to a 9 GHz transmit beacon on NG SLR radar Line of Sight

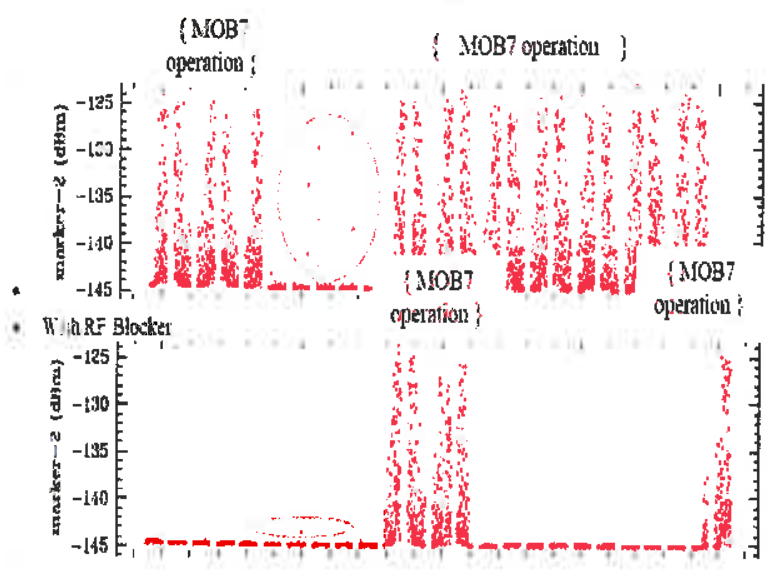

Figure 3: An RF Blocker was strategically inserted between the NG SLR radar antenna and the VLBI anterna at GGAO
An RF blocking wall is being strategically planned to lessen the requirement for these masks. The blue circles on the plots in Figure 3 show the potential effect of a blocker that was inserted between the radar used for the NG SLR and the VLBI antenna. That day the MOB7 radar was not blocked at all but when it was off 10-20 dB less radar signal was detected.

As Figure 4 illustrates, even for a single baseline there is a large hole in the observable sky for VLBI antennas having to avoid the SLR radar.

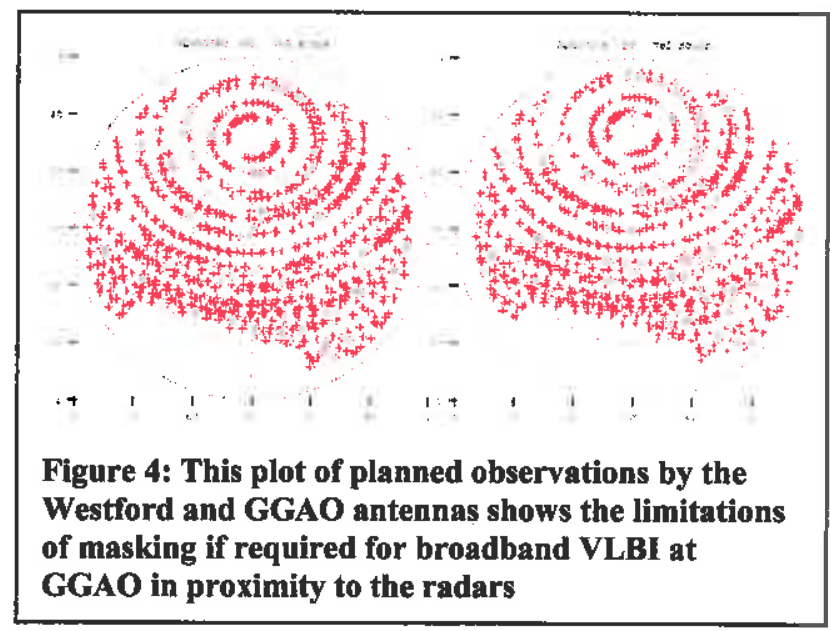

\section{DORIS RFI STRATEGY}

Because DORIS has a hard requirement to be hemiomni, masking is not a relevant strategy for RFI mitigation. DORIS operates at 2036.25 $\mathrm{MHz}$ and 401.25 $\mathrm{MHz}$ so it achieves the desired 5:1 ratio for Doppler ranging. Filtering already implemented on the VLBI antenna that separates the low band from the high band for separate gain management and fiber optic isolation, will be good strategy at most new broadband stations.

For the well-behaved DORIS, notch filtering in the VLBI receiver is more likely to work than an RFI 
blocking wall that may produce multipath. An RF trap of absorber material and reflector material in a Salisbury screen has also been investigated. At GGAO, the present DORIS site is not a problem because it is over 200 meters from VLBI and behind several buildings on the GGAO campus. That isn't the case at all geodetic test sites, so a recommendation for DORIS-VLBI distances that are close, but not too close, to each other will be made by the Space Geodesy Project. Research is underway at GGAO to characterize a spare DORIS beacon at slightly different frequency so as not to confuse the ranging receivers in the DORIS constellation.

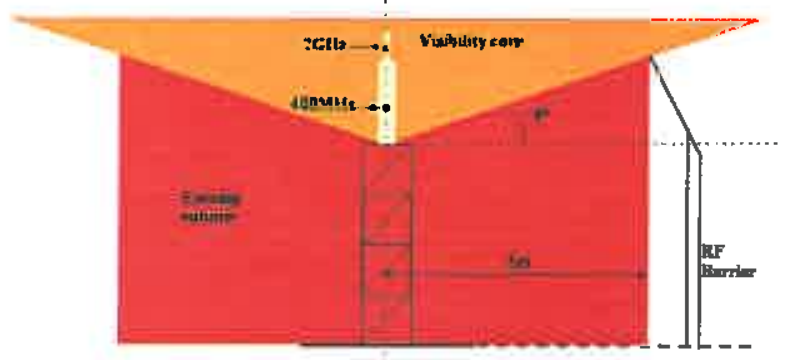

Figure 5: The DORIS Near Field environment has to be carefully maintained to design RFI mitigation. At GGAO, the layout provides enough path loss to have both techniques coexist

A super stable time and frequency reference such as the VLBI maser is important to all of the techniques. Also important is a good monitoring system that surveys the stability of all the techniques. The vector tie system will tie together pointing references such as phase center and physical survey landmarks. Meteorological sensors need to be at every sight to independently measure pressure which affects wave propagation. The Monitor and Control Infrastructure (MCI) monitors critical voltages, currents, and temperatures and pressure is first being developed for the VLBI 2010 stations but a version of MCI is also relevant for SLR. Fellow broadband developers at Wetzell and Munich Germany have developed a framework for multi-station monitoring, Haystack Observatory is developing software for the node computer in the GGAO front end,

The SGP project has also commissioned site surveys that look at all critical factors to consider when adding one or more techniques to a site. Many of the recommendations from this study will have to be adapted to local work schedules and the presence of RFI.

For SLR, cloud cover is an important consideration. For VLBI, the southern hemisphere is largely uncovered, so NASA's SGP may be technical consultants and funding impetus that produces a world-wide geodetic network of multi-technique and long term measurements of climate change.

\section{REFERENCES}

[1] Beaudoin, C., Corey, B.E., Hilliard, L, Petrachenko, B., (2012) RF compatibility of VLBI with DORIS and SLR at GGOS stations: An experimental methodology to validate the models, International VLBI Service for Geodesy and Astrometry 2012 General Meeting Proceedings, March. 4-9, 2012, Madrid, Spain, (in-press).

[2] Hilliard, L.M. , "Space Geodesy Project (SGP) Colocation considerations and Radio Frequency Interference (RFI) Mitigation Techniques", International DORIS Service Workshop at the 20 years of Progress in Radar Altimetry" Symposium in Venice-Lido, Italy 24 - 29 September, 2012

[3] Tourain, C. 2012, "Temporary DORIS installation for VLBI / DORIS compatibility tests", Technical Memo, Nov. 12, 2012 